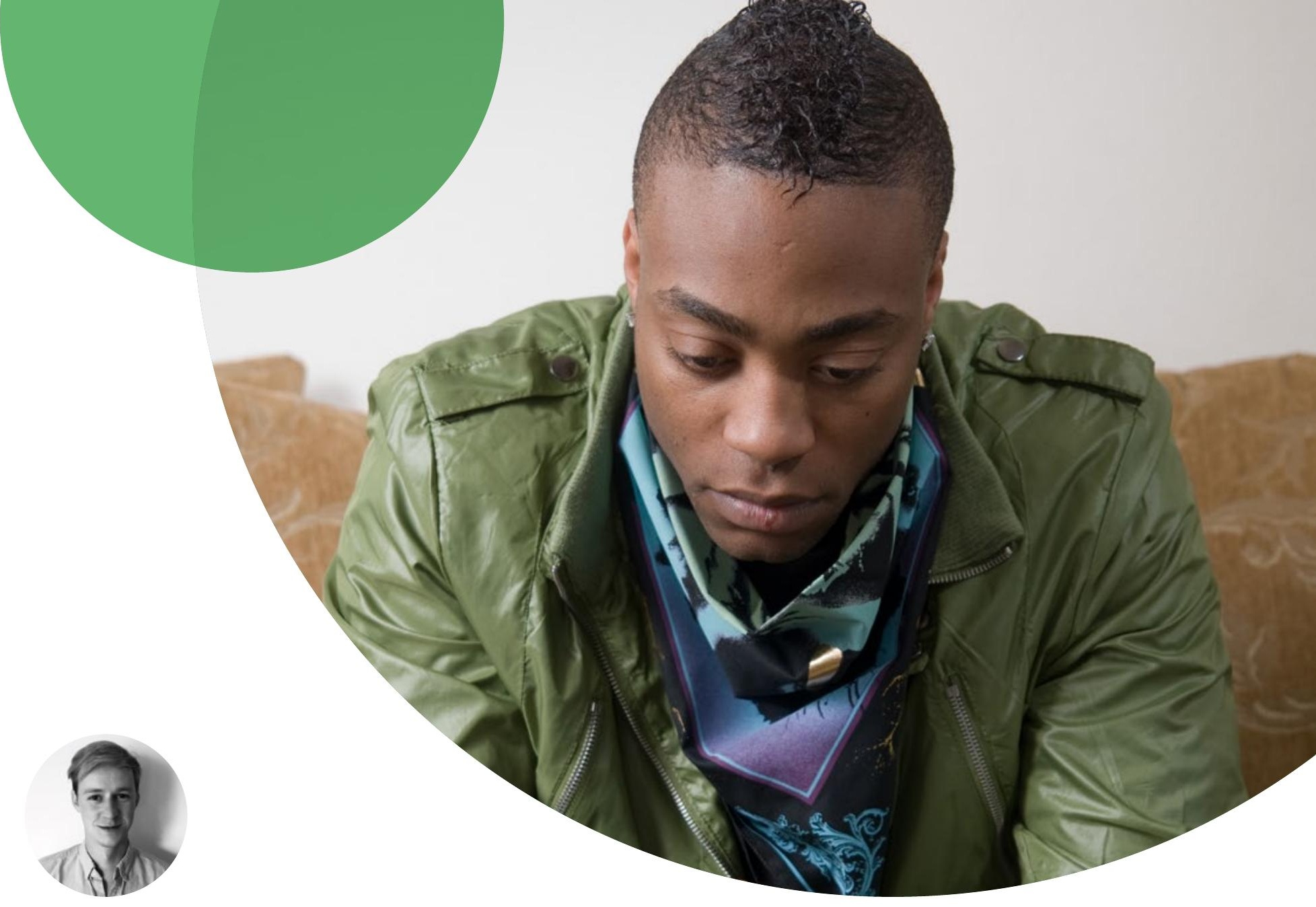

\title{
Continued family dysfunction accounts for the association between childhood adversity and adolescent self-harm
}

By Matthew Cassels

Matthew Cassels has a PhD in Child and Adolescent Psychiatry and an MPhil in Social and Developmental Psychology from the University of Cambridge where he specialized in identifying risk factors and correlates of non-suicidal self-injury among adolescents. Matthew's doctoral studies were funded by a Gates Cambridge Scholarship and the present study was funded by the Wellcome Trust.

This article is a summary of the paper published in JCPP - Cassels M, van Harmelen A-L, Neufeld S, Goodyer I, Jones PB, Wilkinson P. (2018). Poor family functioning mediates the link between childhood adversity and adolescent nonsuicidal self-injury. Journal of Child Psychology and Psychiatry, 59(8), 881-88, doi: 10.1111/jcpp.12866

Non-suicidal self-injury (NSSI) is any deliberate attempt at inflicting physical self-harm in the absence of suicidal intent. NSSI peaks during adolescence, with roughly $17 \%$ of adolescents reporting that they have engaged in it at least once. NSSI is predictive of mental health problems and suicide attempts, and is associated with numerous other negative social, psychological, and physical outcomes. As such, identifying possible risk factors and targets for intervention of NSSI is of paramount importance. Childhood family adversity (CFA) has been identified as one of the major risk factors for
NSSI during adolescence. However, the ways in which traumatic events that occur in early childhood confer risk for the emergence of NSSI up to a decade later are unclear. Clarifying these pathways could help inform specific treatment, intervention, and risk prevention targets for adolescent NSSI.

In a new study published in the Journal of Child Psychology and Psychiatry, we tested four alternative hypotheses to explain this association between CFA occurring before age 5 and new onset of NSSI between ages 14 to 17 . 
1) Mental illness: CFA is a strong predictor of increased mental illness. Nearly all mental illness is, in turn, associated with increased rates of NSSI. Therefore, adolescent NSSI may arise from mental illness resulting from CFA as opposed to being a direct result of the original CFA. In this case, interventions could focus on addressing mental illness.

2) Continued family dysfunction: CFA can have a lasting negative impact on family functioning and childparent relationships, and it often occurs within a wider context of continuing family dysfunction. This continued family dysfunction associated with CFA may act as a proximal risk for NSSI as opposed to the CFA itself. In this case, interventions aimed at improving family dysfunction may be most effective at reducing NSSI.

3) Social buffer: Positive family and/or peer relationships in adolescence may buffer the association between CFA and NSSI, either because social support acts as a protective factor, or because the family members who previously contributed to adversity are now more positive influences.

4) Attachment: Conversely, attachment theory suggests that early experiences have a lasting and immutable impact on emotional development and psychopathology. Thus, CFA may be a necessary and sufficient cause for NSSI regardless of more proximal factors. In this case, the most effective measure for reducing adolescent NSSI may be focusing on preventing CFA.

To test these four possible pathways, data were collected from a community sample of 933 fourteen year-olds with no history of NSSI. At baseline (age 14), data were collected on lifetime history of NSSI and diagnosis of mental illness, CFA occurring before age 5 , as well as current family functioning, quality of friendships, and socioeconomic status. Follow-up data on NSSI were collected again three years later (age 17). As the sample was restricted to participants who reported no lifetime NSSI at age 14, all instances of NSSI reported at age 17 occurred after baseline data collection. Thus, all observed associations between other variables and NSSI are prospective.

A total of 59/933 (6\%) participants reported new onset of NSSI by age 17. Poorer family functioning at age 14, mental illness by 14, and CFA before age 5 were all significant risk factors for NSSI by age 17. Friendships, socioeconomic status, and gender were not associated with NSSI. Path analyses supported the continued family dysfunction model; the association between CFA before age 5 and subsequent onset of NSSI between 14-17 years was largely accounted for by continued poor family functioning at age 14 .

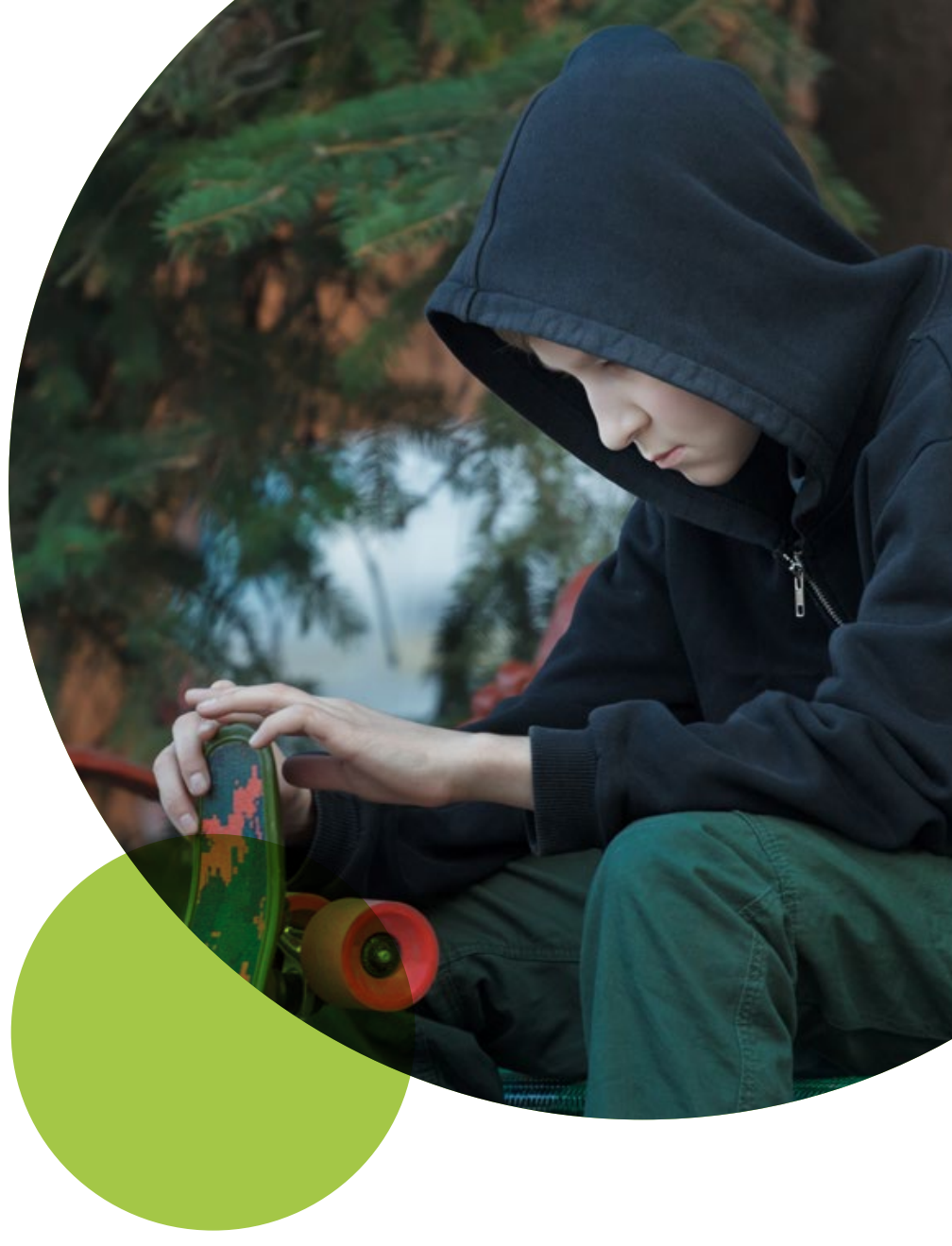

When traumatic experiences happen in the context of continuing family dysfunction, are perpetrated by a parent figure, or are not responded to adequately by a parent, family functioning is likely to be impaired. Impaired family functioning later in adolescence is, in turn, robustly associated with NSSI. The present findings, however, suggest that improving family relationships may reduce the risk of NSSI among children who have been exposed to CFA.

Our analyses did not support any of the other hypothesized pathways from CFA to NSSI. Specifically, the pathway from CFA to NSSI through mental illness was not significant, and we found no support for the protective effects of positive peer or family relationships. In fact, peer relationships were not associated with NSSI in any way, in keeping with the literature. With regard to family relationships, whether or not family dysfunction continues seems to be the primary factor that influences risk of NSSI. Furthermore, we found no support for the attachment model; there was no significant direct association between CFA and NSSI when proximal family functioning and mental illness were accounted for. This suggests that the effects of CFA on NSSI are modifiable and perhaps that internal working models of threat can be updated by subsequent experience. Although further research is needed to investigate potential methods of 
improving family relationships after CFA, the present findings support family-focused approaches to preventing adolescent NSSI. The findings also highlight the importance that services helping families in trouble, such as social care, try to address family relationships directly.

One limitation of this study is that NSSI was measured with a single-item, 'Have you ever tried to hurt yourself on purpose without trying to kill yourself?' This may not have been sufficient to capture all NSSI acts and did not distinguish between different methods, motivations or frequencies of NSSI. This is potentially problematic as different methods and frequencies of NSSI have been related to different psychological and environmental factors. Another limitation of this study is that the sampling age range may have been too late to capture many first incidents of NSSI. The natural course of NSSI is curvilinear, with a sharp increase around age 12 and a decrease in later adolescence. Longitudinal studies beginning at a younger age (before 12) would be greatly beneficial as they would capture more first incidents of NSSI.

Despite these limitations, the present study showed that the well-demonstrated association between CFA and adolescent NSSI can largely be accounted for by continued family dysfunction, supporting a family focused approach to NSSI treatment and intervention.
Key points:

\section{Overview}

- Childhood family adversity (CFA) is a welldemonstrated risk factor for adolescent nonsuicidal self-injury (NSSI).

- Pathways between CFA and subsequent onset of NSSI are unclear.

-We demonstrate that the association between CFA before age 5 and NSSI between ages 1417 is due in large part to continued poor family functioning at age 14 .

\section{Clinical practice}

- These findings suggest that improving family function after CFA may prevent later NSSI.

- These findings support a family-focused approach to preventing adolescent NSSI.

\section{Gaps and recommendations for further science}

- Future research should explore the efficacy of family focused therapies for reducing incidence of NSSI among young people with histories of CFA.

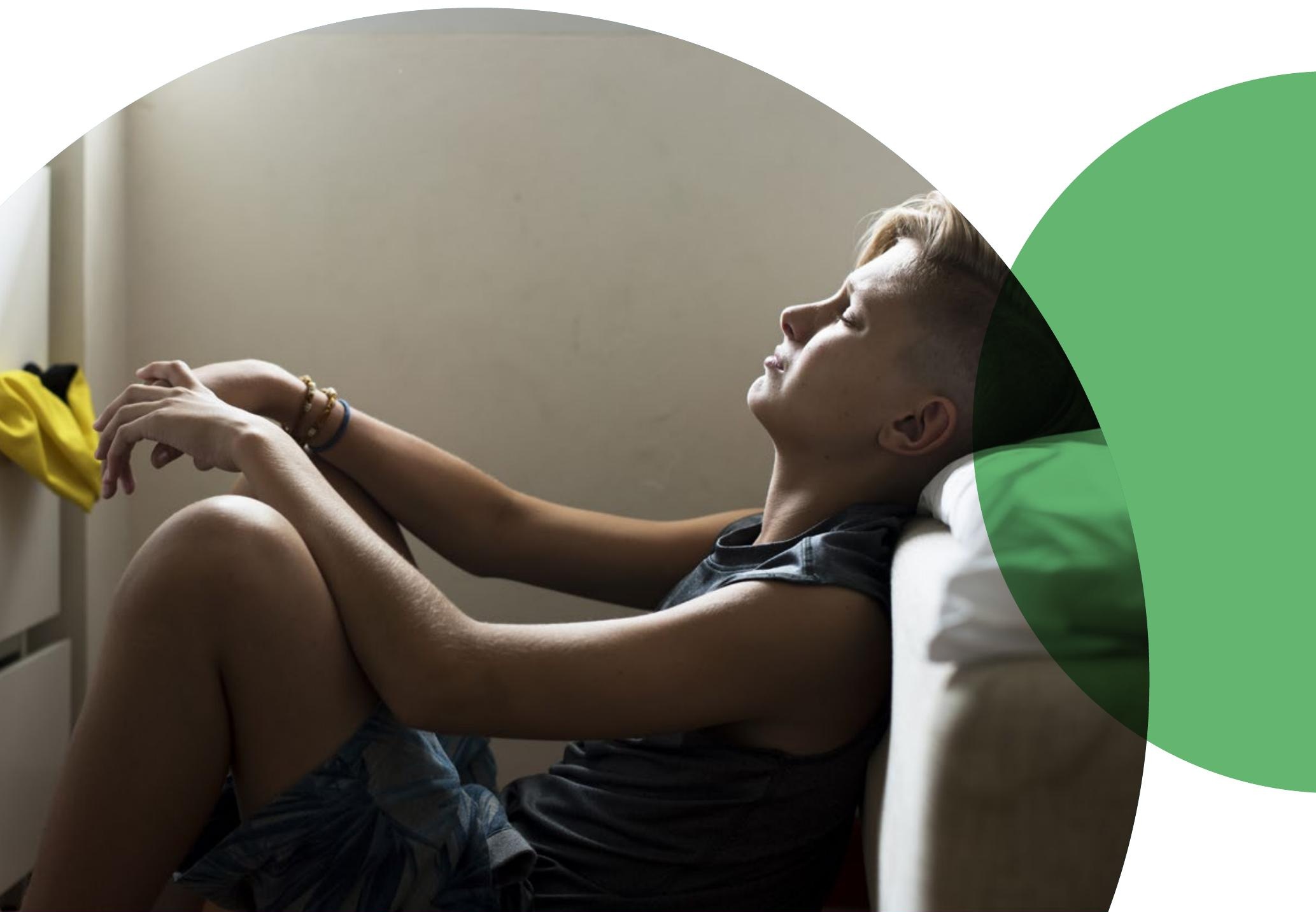

